\title{
Editorial
}

\section{Stent magic! The genie has escaped from the bottle}

A response to injury leading to intimal thickening and subsequent "restenosis" has limited the clinical efficiency of percutaneous coronary interventions ever since they have been used to treat symptomatic coronary artery disease. During the recent two decades a lot has been learned about the pathophysiology of the response to injury following different kinds of interventions. The predominant mechanism is activation of cells within the arterial wall, the adventitial layer, and the blood, namely arterial smooth muscle cells, fibroblasts, endothelial cells, monocytes, lymphocytes, and leucocytes. Once activated by arterial injury, most of these cells will undergo a change of phenotype and express a variety of growth factors and hormones leading to complex interactions, which result in cellular migration and proliferation. As a result, much research has focused on modulation or inhibition of cell proliferation and, although some compounds were effective in animal models, they were not successful in human restenosis trials.

One explanation for this failure is a lack of appropriate animal models, with species related specific differences in cell activation and cellular responses, which negates comparison with human atherosclerotic coronary arteries. Nevertheless, we have learned from this experimental work that the response to injury is rather specific for each kind of injury. The time course might be quite different-for example, a short burst of proliferation ensues after conventional balloon angioplasty, while a long lasting proliferative response is induced by implantation of metallic stents. Multiple attempts had been made to ameliorate the arterial response to injury by using different mechanical techniques for ablating or debulking atherosclerotic tissue before dilatation of the vessel was performed. Although attractive in theory, these techniques, like excimer laser angioplasty and directional or rotational atherectomy, have not influenced restenosis rates and hence have not led to a major change in practice in the interventional catheterisation laboratories. By contrast, a technique which has had a tremendous impact during recent years is the implantation of stents, despite the well known experimental data showing, in nearly all animal species, that no other technique is better at provoking a response to injury and neointimal thickening. ${ }^{1}$

\section{Vessel wall response to stenting}

The arterial vessel wall response following stent implantation is predominantly a result of exaggerated cellular migration, proliferation, and expression of extracellular matrix constituents by vascular smooth muscle cells. Once it has occurred, this process is difficult to reverse, not only in experimental animals models but also in human coronary arteries, when compared to restenosis following balloon angioplasty. These difficulties have spawned a generation of cardio-radio-oncologists, using $\beta$ or $\gamma$ radiation to inhibit cellular activation. Radiation, however, is difficult to handle and does have side effects, leading to cell necrosis and late vessel ulceration and ultimate thrombosis. ${ }^{2}$ Locally delivered antimitotic compounds-for example, paclitaxel-might help in this situation; however, further studies are needed with either stent based or other local drug delivery devices. ${ }^{3}$

In the mid 1990s the policy for percutaneous interventions started to swing more and more towards the use of stents, their use being easier and placement more successful owing to the considerable improvements in design and flexibility. However, available data on the success of stents to reduce restenosis remained scientifically doubtful and inconclusive. The BENESTENT I and STRESS trials demonstrated, in a carefully defined group of lesions, a marginal advantage of $0.18 \mathrm{~mm}$ in average minimal lumen diameter towards stenting compared with conventional balloon angioplasty, at six month angiographic follow up. These trials have led to widespread and almost indiscriminate use of stents in a wide range of lesions. Indeed, the new millennium has started in a flair of "stentomania". In this situation, the WIDEST study was timely and scientifically necessary. ${ }^{4}$ The authors must be congratulated on their efforts to perform a trial comparing restenosis after "optimised" balloon angioplasty or Wiktor stent implantation. The statement and rationale that "the rapid expansion in the use of coronary stents has not been supported by adequate data" was not only true some five years ago but is still arguably the major problem in interventional cardiology. The results of the WIDEST trial indicate that a strategy of provisional stenting, which means an optimal luminal size after balloon angioplasty, favourably compares to elective stenting. This finding is not surprising since not only improvement in stent design but also refinements in the technique of balloon angioplasty have been achieved during the recent years. So what is it that keeps the whole interventional world believing that the best solution for treatment of atherosclerotic coronary artery disease is the implantation of coronary stents?

\section{Angiographic appearance after stenting}

One of the most important reasons might be the angiographic appearance of the target site after stenting which most often is smooth looking when compared to the result after conventional balloon angioplasty which, in the majority of patients, shows some haziness, irregular vessel contours, and dissections. So, are we casualties of an oculo-motoric reflex rather than staying more sophisticated? Yes, probably we are, since, as shown by the data from the ESPRIT trial, which has been reported recently, the complication rate associated with elective stenting in the acute setting is about $10 \%$ with regard to death, myocardial infarction, and urgent target vessel revascularisation. ${ }^{5}$ Furthermore, it has been shown

Trial acronyms

BENESTENT: Belgium-Netherlands Stent Study

ESPRIT: Enhanced Suppression of the Platelet GPIIb/

IIIa Receptor with Integrilin Therapy

STRESS: Stent Restenosis Study

TIMI: Thrombolysis in Myocardial Infarction

WIDEST: Wiktor Stent in De Novo Stenosis 
conclusively that prognosis of patients undergoing interventions strongly depends on the incidence and magnitude of minor or major myocardial necrosis. ${ }^{6}$ Stenting is associated with an increased rate of myocardial injury when compared to conventional balloon angioplasty, and this most probably results from extrusion of atherosclerotic tissue leading to an increased number of small distal emboli. ${ }^{7}$ Thus, as most often in life, there is a price to pay for a nice looking result. This is not only the budget price for the individual stent but also a higher rate of acute events, and an impairment of prognosis, in some of our patients.

In the WIDEST trial, at six months follow up, both the angiographic and clinical results were essentially identical in patients treated by either balloon angioplasty or stenting. One might argue that the balloon angioplasty group was assisted by the crossover rate of some $30 \%$. However, these results have to be seen also in the light of previously performed studies which showed very similar results when late clinical outcome was compared - that is, BENESTENT II and STRESS. If the long term clinical outcome remains comparable between patients treated with conventional balloon angioplasty or coronary stenting, the question arises again why stenting is still, in practice, the treatment of choice in up to $85 \%$ of our patients. Based on the WIDEST trial, clinical data justifying this high rate of stent usage is substantially lacking.

Use of stents has clearly enriched our armamentarium in the catheter laboratory in that it allows more "aggressively" optimised balloon angioplasty, achieving the best luminal result and coronary flow even if dissections might occur. Dissections per se might even be beneficial for the vascular healing process if flow is normal by angiographic criteria (TIMI III). This suggests another approach to stent usage, which is perhaps best described as "conservative" stenting. Presently there are only anecdotal reports to support this. ${ }^{89}$ A randomised trial would be of major interest scientifically and, of course, economically. Reluctance to finance such trials by industrial grants is understandable. However, public financing for such a trial must be found if we are serious about practising evidence based medicine.

However, is the climate for such a trial now impossibly hostile? Do the vast majority of interventional cardiologists indeed prefer to believe what they have been told about nice looking angiographic pictures, rather than listening to biologically founded arguments? Problems with the WIDEST study itself suggest this may be so. Although the study was originally aimed at 400 patients, it was stopped prematurely after enrolment of 300 patients because of declining recruitment rates. These most probably reflected the change in interventional cardiology, implanting more and more stents and judging the quality of an interventional centre simply by the rate of stent implantation.

Just as angioplasty has led to the new problem of "restenosis", the emergence of stenting, like a genie from the bottle, has led to the new and even more intractable problem of "in-stent restenosis". Surely-and the WIDEST trial demonstrates this - it is time to consider curbing the indiscriminate use of stenting, so that the benefits in terms of safer balloon angioplasty can outweigh the costs and potential harm. Unfortunately, now that the stenting genie is out of the bottle, it may be almost impossible to mount a trial to determine its true value.

K R KARSCH A C NEWBY

Bristol Heart Institute,

University of Bristol,

Bristol Royal Infirmary,

Bristol, UK

email:K.R.Karsch@bristol.ac.uk
1 Hanke H, Kamenz J, Hassenstein S, et al. Prolonged proliferative response of smooth muscle cells after experimental intravascular stenting. Eur Heart $\mathcal{F}$ 1995;16:785-93.

2 Costa MA, Sabate M, van der Giessen WJ, et al. Late coronary occlusion after intracoronary brachytherapy. Circulation 1999;100:789-92.

3 Axel D, Kunert W, Goggelmann CH, et al. Paclitaxel inhibits arterial smooth muscle cell proliferation and migration in vitro and in vivo using local drug delivery. Circulation 1997;96:636-45.

4 Fluck DS, Chenu P, Mills P, et al, for the WIDEST Trial Investigators' Group. Is provisional stenting the effective option? The WIDEST study (Wiktor stent in de novo stenosis). Heart 2000;84:522-8.

5 Tcheng JE. The ESPRIT Trial. ACC Anaheim 2000, Trial Hotline, oral presentation.

6 Topol EJ, Ferguson JJ, Weisman HF, et al. Long-term protection from myocardial ischaemic events in a randomised trial of brief integrin beta (3) blockade with percutaneous coronary intervention. FAMA 1997;278:47984

7 Garbaz E, Jung B, Lefevre G, et al. Frequency and prognostic value of cardiac troponin I elevation after coronary stenting. Am $f$ Cardiol 1999;84:515-18

8 Schroder S, Baumbach A, Mahrholdt $\mathrm{H}$, et al. The impact of untreated coronary dissections on acute and long-term outcome after intravascular ultrasound guided PTCA. Eur Heart f 2000;21:137-45.

9 Cappelletti A, Margonato A, Rosano G, et al. Short- and long-term evolution of unstented non-occlusive coronary dissection after coronary angioplasty. F Am Coll Cardiol 1999;34:1484-8.

\section{WEB TOP 10}

www.heartjnl.com

These articles scored the most hits on Heart's web site during August 2000

\section{The diagnosis of heart failure}

A D Struthers

September 2000;84:334-8 (Education in Heart)

2 Timing of aortic valve surgery

C M Otto

August 2000;84:211-18 (Education in Heart)

3 Treatment of atrial flutter

A L Waldo

August 2000;84:227-232 (Education in Heart)

4 Joint British recommendations on prevention of coronary heart disease in clinical practice

December 1998;80(suppl 2):S1-29

5 Antiarrhythmic drugs: from mechanisms to clinical practice

D M Roden

September 2000;84:339-46 (Education in Heart)

6 The impact of time to thrombolytic treatment on outcome in patients with acute myocardial infarction P Chareonthaitawee, R J Gibbons, R S Roberts, T F Christian, R Burns

August 2000;84:142-8 (Cardiovascular medicine)

7 Treatment of cardiac diseases: evidence based or experience based medicine?

W Kubler

August 2000;84:134-6 (Review)

8 Identifying failure to achieve complete (TIMI 3) reperfusion following thrombolytic treatment: how to do it, when to do it, and why it's all worth doing K G Oldroyd

August 2000;84:113-15 (Editorial)

9 Low dose amiodarone and sotalol in the treatment of recurrent, symptomatic atrial fibrillation: a comparative, placebo controlled study

G E Kochiadakis, N E Igoumenidis, M E Marketou, M D Kaleboubas, E N Simantirakis, P E Vardas

September 2000;84:251-7 (Cardiovascular medicine)

10 Failure of thrombolysis by streptokinase: detection with a simple electrocardiographic method

A G C Sutton, P G Campbell, D J A Price, E D Grech, J A

Hall, A Davies, $M$ J Stewart, $M$ A de Belder

Aug 2000;84:149-56 (Cardiovascular medicine) 